\title{
Modelo de gestión empresarial asociativo para PROACHIRKO, comunidad de Huanangui, Perú
}

\author{
Eduardo Buse Thorne* \\ Mario Chong ChONG** \\ Juan Carlos Mathews Salazar ***
}

\section{RESUMEN}

La presente investigación se realizó para los Productores Asociados de Chirimoya de Kalidad Óptima (PROACHIRKO); asociación agrícola creada en el año 2009 y constituida por cincuenta socios, ubicada en el distrito de Leoncio Prado, provincia de Huaura, Región Lima. Se dedica al cultivo y cosecha de la chirimoya en diferentes condiciones naturales. La investigación tiene como finalidad desarrollar un plan de acción basado en una propuesta de modelo de gestión empresarial asociativo que contemple la participación articulada de los sectores público, privado y académico, conjuntamente con una integración total basada en la confianza, sin embargo, deben mejorar su competitividad y ampliar su oferta en el mercado global. Mediante la investigación se analizará el entorno de PROACHIRKO, se identificarán sus características productivas, administrativas, aspectos asociativos, entre otros, para proponer el modelo que les permitirá garantizar su permanencia en el mercado local y capitalizar el entorno comercial favorable existente para las exportaciones

Palabras clave: chirimoya, confianza, modelo, PROACHIRKO

AsSOCiATIVE BUSINESS MANAGEMENT MODEL FOR PROACHIRKO, Huanangui Community, Peru

\section{ABSTRAC}

This research was conducted for Productores Asociados de Chirimoya de Kalidad Óptima (PROACHIRKO); agricultural association created in 2009 with fifty partners, located in the district of Leoncio Prado province of Huaura, Lima Region. They are dedicated to the cultivation and harvesting of cherimoya in different natural conditions. The research aims to develop an action plan based on a proposed model of business management partnership that includes the coordinated participation of public, private and academic sectors together, with an integration based on trust; however, competitiveness should improve and expand its offerings in the global market. Through research PROACHIRKO will be analyzed: their productive, administrative, associative aspects characteristics are identified, among others, to propose a model that will allow them to ensure their permanence in the local market and capitalize on the existing favorable business environment for exports.

Keywords: cherimoya, trust model, PROACHIRKO

\section{INTRODUCCIÓN}

La investigación se realizó porque PROACHIRKO necesita contar con un sistema de producción eficiente; con mecanismos de representatividad gremial; con un modelo de gestión empresarial asociativo que le permita acceder a fondos privados, estatales y/o internacionales; con un plan de desarrollo para el acceso a los mercados internacionales y el fortalecimiento del mercado nacional.

Lamentablemente, al no cumplir con los fines mencionados durante los últimos cinco años, PROACHIRKO ha crecido de manera desordenada y con un modelo de negocio informal, ocasionando, entre otros problemas, la disminución del número de socios; actualmente treinta cuatro (34) socios activos. Necesariamente se debe desarrollar un plan de acción que recoja mediante instrumentos de investigación, los procesos a mejorar e incorporarlos en una propuesta de modelo de gestión empresarial asociativo.

PROACHIRKO se distingue competitivamente gracias al manejo agronómico de la chirimoya tipo Cumbe, en la que utiliza la técnica de poda y polinización asistida que fuera proporcionada por el Instituto Nacional de Innovación Agraria (INIA) y por consultores chilenos contratados por la asociación. La técnica de polinización asistida consiste en la floración uniforme de la planta, por la aplicación manual del polen en cada flor. Adicionalmente están incursionando en riego tecnificado con 18 hectáreas y en la certificación GLOBALG.A.P. con ocho (08) hectáreas.

La venta y entrega del producto se realiza de setiembre a diciembre (meses de contra-estación), ya que en estos meses tienen la capacidad de negociar en volumen. El precio promedio anual de la chirimoya de PROACHIRKO es $50 \%$ mayor al precio promedio del mercado. Su principal cliente es YRLOSA SAC, mayorista de chirimoya en Lima y representa el $90 \%$ de sus ventas.

El producto se entrega en el campo, en cajas de veinte kilogramos, envueltas con papel de estraza o craft. El comprador asume

\footnotetext{
Eduardo Buse Thorne, PhD Gestión de Empresas por la UNMSM, docente en la Universidad del Pacífico, gerente general de Quo Vadis Consultores Asociados. E-mail: ew.buset@up.edu.pe

** Mario Chong Chong, PhD Gestión de Empresas por la UNMSM, corporativo y director de Programas Internacionales en la Universidad del Pacífico. E-mal: m.chong@up.edu.pe

*** Juan Carlos Mathews Salazar, licenciado en Economía, Economía y Administración por la Universidad del Pacífico, director Maestría Negocios Globales en la Universidad del Pacífico. E-mail: mathews_jc@up.edu.pe
} 
la logística de salida del producto, usualmente el mismo día de la cosecha.

\section{Objetivo general}

- Elaborar el plan de acción estratégico para PROACHIRKO en la comunidad campesina de Huanangui (distrito de Leoncio Prado, provincia de Huaura, departamento de Lima) y su articulación al mercado nacional e internacional.

\section{Objetivos específicos}

- Analizar el entorno de PROACHIRKO.

- Evaluar el planeamiento estratégico vigente de PROACHIRKO.

- Identificar y segmentar el mercado.

- Desarrollar los planes de funciones: marketing, operaciones, recursos humanos y financieros.

- Incorporar un modelo de desarrollo que contemple la asociatividad basado en la confianza

\section{MARCO TEÓRICO}

\section{Producto}

La chirimoya, Annona Cherimola, pertenece a la familia de las Anonáceas. El tiempo de maduración fisiológica por polinización asistida es de ocho años y su período de explotación dura quince campañas agrícolas o quince años, luego el campo pasa por un proceso de renovación del cultivo.

\section{Los ecotipos de este fruto son:}

- Impresa: forma cónica, globosa, superficie de la cáscara con escamas ligeramente pronunciada, es conocida también como "Blanca de Huanangui".

- Lisa: fruta sin protuberancias forma corazonada globosa, es conocida como "Cumbe".

- Tuberculata o criolla: forma cónica con protuberancias escamas muy pronunciadas.

El ecotipo más comercial es la Cumbe. La fruta posee una pulpa cremosa, ligeramente grumosa de aspecto gelatinoso con semillas de color negro brillante, el sabor dulce es similar a la fresa o frambuesa y con un fino aroma a canela. Su principal aporte nutricional (ver Tabla1) es el elevado porcentaje de potasio, ayuda a regular la actividad neuromuscular y promueve el desarrollo celular.
El consumo de esta fruta está indicado para personas con insuficiencia cardíaca, infecciones en el estómago y reduce el nivel de colesterol y ácido úrico.

\section{Cultivo}

La chirimoya se puede cultivar en los valles interandinos de la región Yunga, entre los 1500 y $2000 \mathrm{~m}$ s. n. m., con una clima subtropical y temperaturas entre los 14 y $24^{\circ} \mathrm{C}$, humedad relativa del $80 \%$ y suelos franco arcillosos, es exigente en materia orgánica, pudiéndose lograr rendimientos de hasta 9724 kilos por hectárea (ver Tabla 2).

Los chirimoyos tienen un período vegetativo perenne; la campaña agrícola empieza ocho meses antes de la cosecha, con las podas (de formación, sanidad y rejuvenecimiento) y defoliación del árbol o "agoste". La siguiente etapa es el abonamiento y manejo fitosanitario preventivo, luego recibe el "golpe de agua" que propicia la emisión de brotes (follaje y flores).

La polinización asistida permite la maduración progresiva de la fruta y el período de cosecha puede durar hasta dos meses en la misma planta. Los productores pueden programar sus cosechas en la época de contra-estación (de setiembre a diciembre).

\section{Poscosecha}

El manejo poscosecha tiene los siguientes procesos: retiro de frutas no aptas para la comercialización, selección por tamaño, selección de acuerdo con los requerimientos del cliente, eliminación de los restos de polen, lustrado y encerado con cáscara con cera de uva.

\section{METODOLOGÍA}

La metodología implementada busca incorporar un modelo de gestión empresarial asociativo que contemple distintos aspectos: asociatividad, confianza, participación del sector público, privado y académico, además permite identificar los procesos y establecer las estrategias de mejora en el tiempo, bajo las siguientes interrogantes:

- ¿De qué manera la asociatividad y la confianza generan beneficios a PROACHIRKO?

- ¿Es importante la participación de los otros actores (público, privado y academia) como elementos que fortalecen la cadena de valor de la chirimoya? 
- ¿En qué situación deberá estar PROACHIRKO en cinco años?

- ¿Cómo generará los niveles de rentabilidad para los asociados en cinco años?

- ¿En qué procesos de investigación, desarrollo e innovación $(I+D+i)$ deberá concentrar sus esfuerzos en los próximos cinco años?

- ¿Cómo debería incrementar la productividad y la generación de valor para los clientes?

Las etapas fueron:

1. Documentación de los procesos (encuestas, entrevistas, diagrama de bloques, diagrama de operaciones, diagrama analítico del proceso, recolección de datos).

2. Identificación de los procesos críticos y sus prioridades (lista de verificación, histograma, pareto, matriz de priorización).

3. Análisis de las causas (diagrama causa efecto, lista de verificación, diagrama de dispersión, diagrama de tendencia).

4. Establecer el plan de mejora y el nivel de desempeño (diagrama de control).

5. Medición de los resultados (lista de verificación, diagrama de control).

6. Elaboración del modelo de gestión empresarial asociativo.

\section{Fuentes primarias}

Las fuentes primarias se basaron en entrevistas a profundidad realizadas a opinantes expertos, quienes conocen el tema de la chirimoya en la práctica, en lo académico, en lo profesional y en diferentes aspectos relacionados al fruto. La entrevista a profundidad facilita la recolección de información porque es una herramienta flexible capaz de adaptarse a cualquier condición, situación o personas (ver Tabla 4).

\section{Fuentes secundarias}

Se consideraron fuentes secundarias nacionales y extranjeras fidedignas, estas provinieron de medios como: The World Bank - International Finance Corporation, The Economist - Intelligence Unit, La Compagnie Française d'Assurance pour le Commerce Extérieur, International Trade Centre ITC - Trademap, Ministerio de Comercio Exterior y Turismo (MINCETUR) y la Comisión de Promoción del Perú para la Exportación y el Turismo (PROMPERÚ), Ministerio de Agricultura y Riego
(MINAGRI), Productores Asociados de Chirimoya de Kalidad Óptima (PROACHIRKO).

\section{RESULTADOS ESPERADOS Y POSIBLES IM- PACTOS}

PROACHIRKO ahora es una asociación con 34 miembros que aspiran crecer de manera articulada y armónica. Para ello se implementará el modelo de gestión empresarial asociativo que permite fortalecer a todos los miembros de la asociación y articular con todos los actores de la cadena, además de contar con un representante elegido (Autoridad integradora) por los agricultores que sirve como interlocutor válido generando confianza, atendiendo y solucionando posibles discrepancias internas, así como la permanente comunicación con todos los actores.

Para acceder a los mercados internacionales, estos necesitan de permanente información referida a los procesos de producción, entrega, etc., para lograr la continuidad requerida. El modelo actúa como elemento que permite la articulación permanente entre todos los miembros de la cadena de valor y así lograr el crecimiento estimado sostenido, contemplando una activa participación sin distingo de todos los miembros (hombres y mujeres) y velando por el cuidado de su principal insumo - las tierras - y lograr sostenibilidad en el tiempo.

El análisis de la matriz producto/mercado de Ansoff (ver Tabla 3) para PROACHIRKO, propone una estrategia de crecimiento basada en el desarrollo de nuevos mercados (exportadores, supermercados y agroindustriales) sin dejar de atender a los mayoristas y acopiadores de fruta).

El plan estratégico y los planes funcionales, con base en el año 2015, se trabajarán bajo los siguientes supuestos:

- La producción se deberá incrementar en una tasa del $5 \%$ anual hasta el quinto año; por la mejora en rendimientos en campo, la estandarización en el manejo del cultivo, incorporación de pequeñas nuevas áreas de producción, reducción de mermas en campo y procesamiento primario.

- La planta de procesamiento con una capacidad de 800 toneladas se deberá reubicar en Sayán, terreno de PROACHIRKO.

- Las ventas se deberán incrementar paulatinamente hasta un $74 \%$ al quinto año.

- El número de socios y el área productiva se mantendrá en los siguientes cinco años. 
A continuación proponemos el modelo de gestión empresarial asociativo que responde a las inquietudes contempladas anteriormente.

Gráfico 1. Modelo de Gestión Empresarial Asociativo.

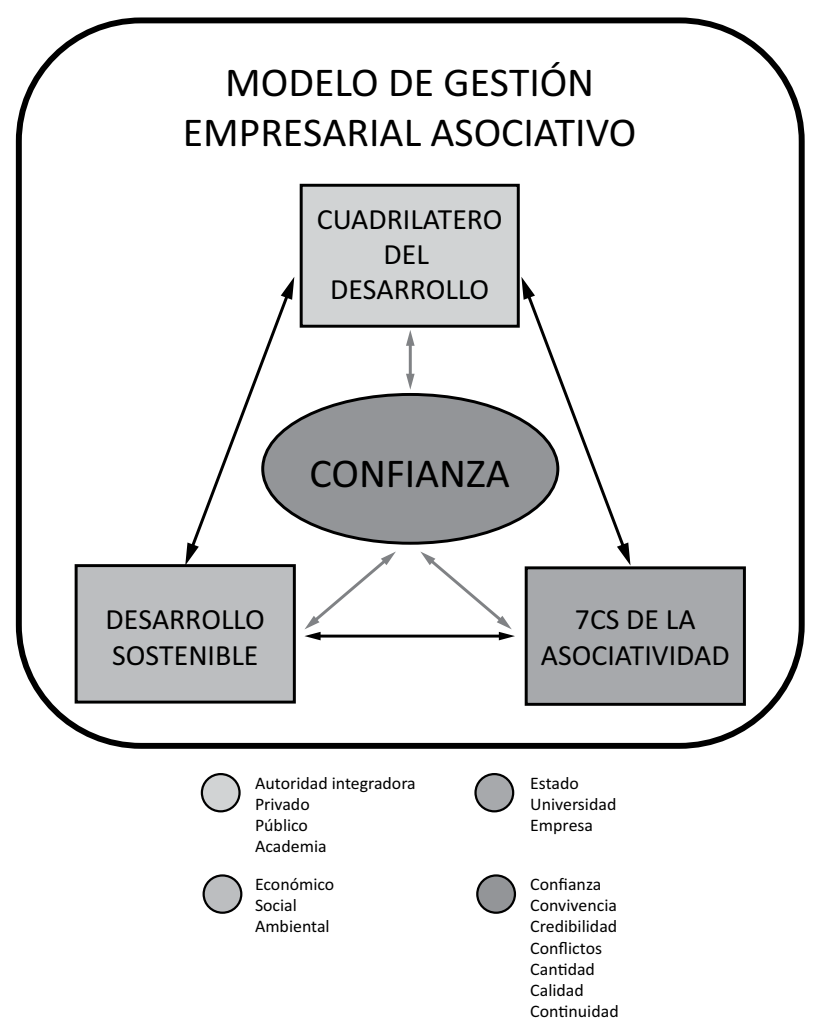

Fuente: Elaboración propia.

\section{CONCLUSIONES}

Para lograr acceder a los mercados internacionales, las pymes necesariamente deben estar asociadas mediante un modelo de gestión empresarial asociativo que les permita articular de manera eficiente los distintos aspectos de la cadena de valor y así competir eficientemente en los mercados. Asimismo generar confianza entre todos y mediante la participación de una autoridad integradora se logra minimizar conflictos.

La participación del sector privado, público y académico son fundamentales para el fortalecimiento de las actividades; el sector privado es el que promueve actividades de desarrollo; el sector público permite viabilizar, con su participación, los requerimientos que el sector privado no puede atender, y el sector académico aporta capacitación y conocimiento para un mejor desempeño del modelo de gestión empresarial asociativo.
El modelo utilizará conceptos como justo a tiempo, calidad total y mantenimiento productivo total como elementos de motivación y participación para la mejora continua.

Finalmente se logra la participación de todos los miembros sin distingo de género o condición social.

\section{REFERENCIAS BIBLIOGRÁFICAS}

[1] Acurio Marmanillo, M. O., Amaya Castillo, J. A., \& Barrantes Caballero, V. M. (2014). Plan de negocios para mejorar la producción y comercialización de chirimoya en la comunidad campesina de Huanangui. Lima.

[2] Banco Mundial. (2015). Sitio web oficial del Banco Mundial. Obtenido de Corporación Financiera Internacional: http://www.ifc.org/ $\mathrm{wps} / \mathrm{wcm} / \mathrm{connect} /$ Multilingual_Ext_Content/ IFC_External_Corporate_Site/Home_ES

[3] COFACE. Sitio web oficial de COFACE. Obtenido de MAJOR MACRO ECONOMIC INDICATORS: http://www.coface.com/ Economic-Studies-and-Country-Risks/Peru (19 de Setiembre de 2014).

[4] Foppiano Rabinovich, G. F. (2013). Formulación y evaluación de proyectos de inversión. Lima: ISIL.

[5] Fred R., D. (2003). Conceptos de Administración Estratégica (11 ed.). México DF: Pearson.

[6] Instituto Nacional de Estadística e Informática. (2015). Resultados finales del IV censo nacional agrario. Lima: INEI.

[7] International Trade Centre. Sitio web oficial de ITC. Obtenido de Trademap: http://www. trademap.org/\%28X\%281\%29S\%28tscpzpzlm yfhxdymthxvnu45\%29\%29/Index.aspx (20 de Mayo de 2015).

[8] International Trade Centre. (2015). Sitio web oficial del ITC. Obtenido de Trademap: http:// www.intracen.org/

[9] La Compagnie Française d'Assurance pour le Commerce Extérieur. (2015). Sitio web oficial de COFACE.

[10] Ministerio de Agricultura y Riego. Sitio web oficial del MINAGRI. Obtenido de Estadística agraria (2013-2014): http://minagri.gob.pe/ portal/ (19 de Setiembre de 2014).

[11] Ministerio de Comercio Exterior y Turismo. (5 de Junio de 2015). Sitio web oficial del 
MINCETUR. Obtenido de Comisión de promoción del Perú para la exportación y el turismo: http://www.mincetur.gob.pe/newweb/

[12] Organización de las Naciones Unidas para la Alimentación y la Agricultura. (Sitio web oficial de la FAO. Obtenido de FAO Statistical Yearbook 2013: http://www.fao.org/docrep/018/ i3107e/i3107e00.htm 10 de Marzo de 2014).
[13] The Economist Intelligence Unit. (2015). Sitio web oficial The EIU. Obtenido de Intelligence Unit: http://www.eiu.com/home.aspx

[14] Universidad Nacional Agraria La Molina. (s.f.). Sitio web oficial de la Universidad Nacional Agraria La Molina. Obtenido de Nuestras Plantas: http://www.lamolina.edu.pe/cventas/ plantas_portada.html

\section{Anexo 1}

Tabla 1. Valor nutricional de la chirimoya

\begin{tabular}{clcc}
\hline Fruta & \multicolumn{1}{c}{ Componente } & Unidad de medida & Por cada $\mathbf{1 0 0} \mathbf{~ g}$ \\
\hline \multirow{6}{*}{ Chirimoya } & Calorías & cal & 81 \\
& Hidratos de carbono & $\mathrm{g}$ & 20 \\
& Fibra & $\mathrm{g}$ & 1,9 \\
& Potasio & $\mathrm{mg}$ & 264 \\
& Magnesio & $\mathrm{mg}$ & 18 \\
& Vitamina C & $\mathrm{mg}$ & 18 \\
& Ácido fólico & micro g & 14 \\
\hline
\end{tabular}

Fuente: Universidad Nacional Agraria La Molina (2015).

Tabla 2. Producción estimada de chirimoya

\begin{tabular}{lcccccc}
\hline Producción de chirimoya & Unidad & Año 1 & Año 2 & Año 3 & Año 4 & Año 5 \\
\hline Kilos $\times$ hectárea & $\mathrm{Kg} / \mathrm{Ha}$ & 8,000 & 8,400 & 8,820 & 9,261 & 9,724 \\
Número de socios & $\mathrm{N}^{\circ}$ & 34.00 & 34.00 & 34.00 & 34.00 & 34.00 \\
Número de hectáreas & $\mathrm{Has}$ & 70.50 & 70.50 & 70.50 & 70.50 & 70.50 \\
\hline Producción total anual & $\mathbf{K g}$ & $\mathbf{5 6 4 , 0 0 0}$ & $\mathbf{5 9 2 , 2 0 0}$ & $\mathbf{6 2 1 , 8 1 0}$ & $\mathbf{6 5 2 , 9 0 1}$ & $\mathbf{6 8 5 , 5 4 6}$ \\
\hline
\end{tabular}

Fuente: Elaboración propia (2014).

Tabla 3. Matriz producto/mercado

\begin{tabular}{llll}
\hline & & Productos existentes & Nuevos \\
\hline \multirow{2}{*}{ Mercados } & Existentes & Penetración de mercados & $\begin{array}{l}\text { Desarrollo de productos } \\
\text { o diversificación }\end{array}$ \\
\cline { 2 - 4 } & Nuevos & Desarrollo de mercados & Diversificación \\
\hline
\end{tabular}

Fuente: H. Igor Ansoff (1957). 


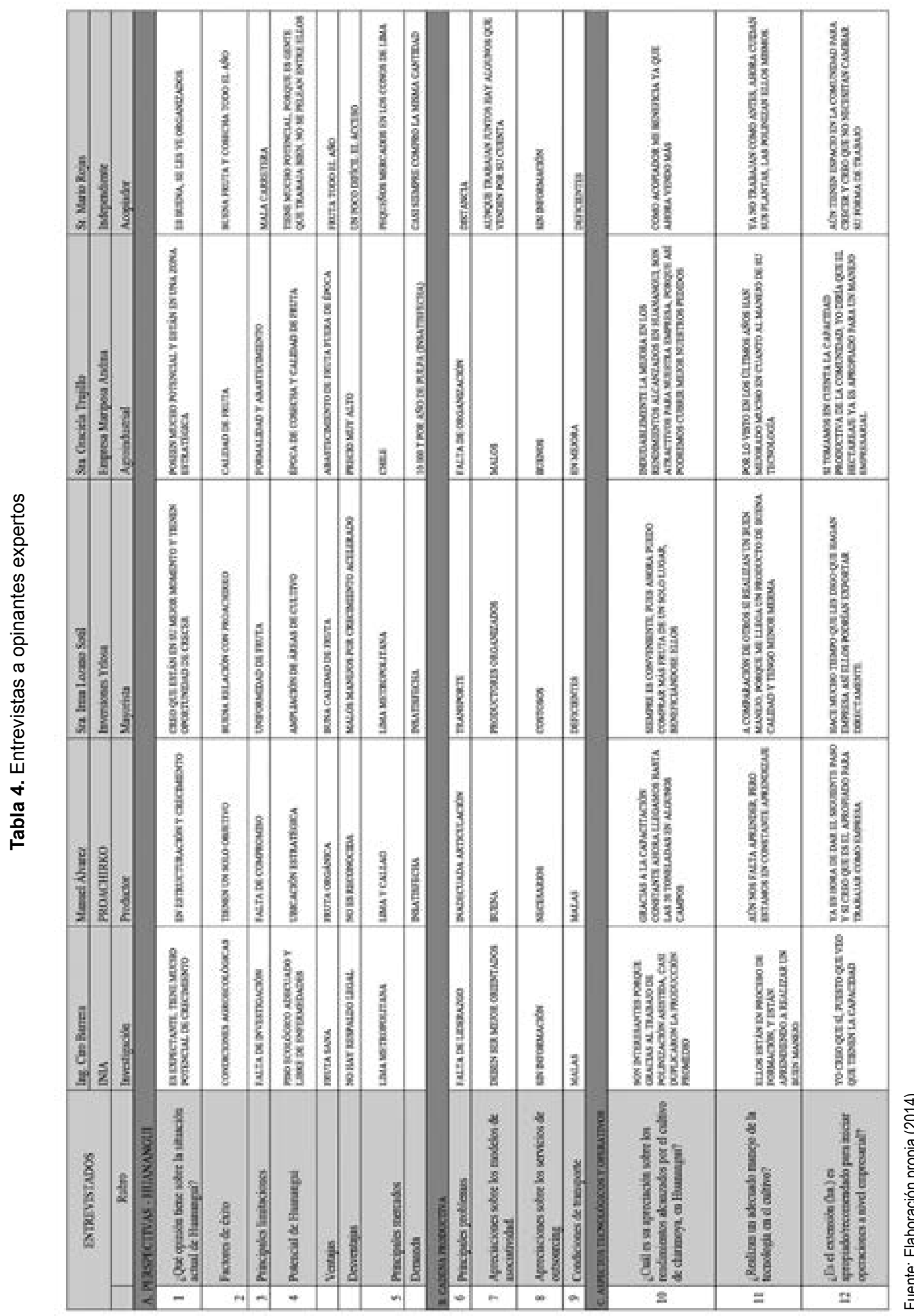

